\title{
Acute promyelocytic leukemia presenting as a paraspinal mass
}

\author{
Nirav N Shah, MD, MSHP, abł Mark Stonecypher, MD, PhD, ${ }^{\text {‘ }}$ Pallavi Gopal, MD, PhD, \\ Selina Luger, MD, ${ }^{\mathrm{ab}}$ Adam Bagg MD, ${ }^{\mathrm{c}}$ and Alexander Perl, $\mathrm{MD}^{\mathrm{ab}}$
}

aAbramson Cancer Center, University of Pennsylvania; ${ }^{b}$ Division of Hematology-Oncology and ${ }^{c}$ Department of Pathology and Laboratory Medicine, Hospital of the University of Pennsylvania, Philadelphia

A cute promyelocytic leukemia (APL) is a distinct subtype of acute myeloid leukemia (AML) that is characterized by a balanced translocation between chromosomes 15 and $17[\mathrm{t}(15 ; 17)]$, which results in the fusion of the promyelocytic leukemia $(P M L)$ and retinoic acid receptor $\alpha(R A R A)$ genes. ${ }^{1,2}$ Historically, APL was a fatal disease because of the high relapse rates with cytotoxic chemotherapy alone and a significant bleeding risk secondary to disseminated intravascular coagulation (DIC). However, APL is now one of the most curable hematological malignancies because of molecularly targeted therapies. ${ }^{1,3}$ With the advent of all-trans retinoic acid (ATRA) containing chemotherapy regimens, rates of complete remission and long-term, disease-free survival have improved dramatically. More recently, regimens incorporating both ATRA and arsenic trioxide (ATO) have allowed a substantial number of patients to be treated with little or no additional cytotoxic chemotherapy, ${ }^{4,5}$

Patients with APL are on average younger than those with non-M3 AML and typically present with cytopenias and signs/symptoms of DIC, such as elevated $\mathrm{D}$-dimer, prolonged clotting times, or decreased fibrinogen., ${ }^{2,3}$ Peripheral smear can demonstrate abnormal promyelocytes containing bundles of Auer rods. ${ }^{2}$ A definitive diagnosis is made by genetic analysis for the $t(15 ; 17)$ in the blood or bone marrow cells. Although extramedullary disease or granulocytic sarcoma is present in $2.5 \%-9.1 \%$ of cases of AML, it is rarely described at initial presentation in patients with APL. ${ }^{6}$ When extramedullary disease does occur in APL, it is seen largely in the setting of relapsed APL and most frequently occurs in the central nervous system which carries with it a poor prognosis. ${ }^{7-9}$ In the present case, we describe a patient who was diagnosed with APL after initially presenting for back pain and was found to have a paraspinal mass.

\section{Case presentation and summary}

A 56-year-old man presented with a 4-month history of back pain. The patient underwent magneticresonance imaging (MRI), which raised concern about a possible neoplastic lesion at T7 (Figure 1). Given the wide differential, a core needle biopsy was performed and the results revealed a necrotic neoplasm of unknown histogenic origin. The patient was referred for neurosurgical evaluation and subsequently underwent T5-T9 decompressive laminectomy with fusion and resection of the epidural mass. Prior to surgery, the patient's white blood cell count was $3,700 / \mu \mathrm{L}$ with an absolute neutrophil count of $2,700 / \mu \mathrm{L}$. His hemoglobin level was $14.9 \mathrm{~g} / \mathrm{dL}$ and his platelet level, $178,000 / \mu \mathrm{L}$. He had no signs of DIC, and the results of his coagulation studies were normal, with a prothrombin time of $12.4 \mathrm{sec}$, international normalized ratio of 1.0 , and partial thromboplastin time of $26.4 \mathrm{sec}$. The patient experienced no postprocedural complications. Preliminary results from the paraspinal mass showed an aggressive hematopoietic tumor and a bone marrow biopsy was performed. The patient felt well and showed no signs/symptoms of disease, so he was discharged pending complete pathological evaluation.

\section{Pathology}

Paraspinal mass. Histologic examination of the paraspinal mass revealed sheets of markedly atypical intermediate-sized cells with round to irregular nuclei, fine chromatin, occasionally prominent nucleoli, and moderate amounts of granular eosinophilic cytoplasm (Figure 2A). Initially there

Accepted for publication August 19, 2015. ¥Authors contributed equally to this work. Correspondence: Nirav N Shah, MD, MSHP; nishah@mcw.edu. Disclosures: The authors report no disclosures or conflicts of interest. JCSO 2016;14:126-129. C2016 Frontline Medical Communications. doi: 10.12788/icso.0220. 
was a broad histologically based differential that included soft tissue sarcomas, numerous poorly differentiated neoplasms, and hematolymphoid malignancies. Once CD45 was shown to be positive and cytokeratin $\mathrm{AE} 1 / 3$ negative, a lymphoma diagnosis was favored; however, the neoplasm expressed neither B- or T-cell antigens. Given those findings, a possible myeloid origin was considered, and subsequent immunohistochemical stains showed that the tumor cells were positive for myeloperoxidase (MPO), CD43, CD45, CD68, and CD117, but negative for all other evaluated antigens (Figure 2B). Taken together, the morphologic and immunophenotypic findings were diagnostic of a granulocytic sarcoma.

Bone marrow biopsy. The Wright-Giemsa stained bone marrow aspirate had features consistent with an acute myeloid leukemia (Figure 3A) with focal collections of

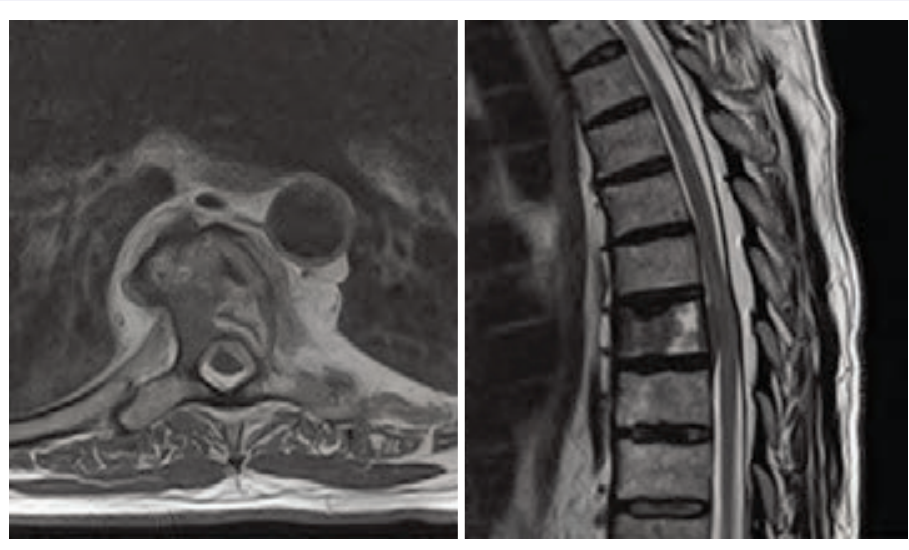

FIGURE 1 Magnetic-resonance imaging of the spine with contrast. Left, axial T1 weighted; Right, sagittal T2 weighted.

"blasts" with round and occasionally bilobed and reniform nuclei, variably condensed chromatin, occasionally prominent nucleoli, and moderate amounts of granular cytoplasm; no Auer rods were identified. An H\&E stained section of the core biopsy showed patchy involvement, with foci of blasts and intervening normal marrow. Flow cytometry on the bone marrow aspirate demonstrated a discrete expanded population of blasts (27\%) with the following dominant immunophenotype: $\mathrm{CD} 13+\mathrm{CD} 33+\mathrm{CD} 64+\mathrm{CD} 117+\mathrm{MPO}+$ HLA-DR- with dim variable expression of CD2, CD4, and CD34.

Metaphase cytogenetics on the bone marrow demonstrated 46,XY,t(15;17)(q24;q21) [15]/46,XY[5] (Figure 3B). Both FISH (fluorescent in situ hybridization) and RT-PCR (reverse transcription-polymerase chain reaction) showed a PML-RARA fusion. A FLT3 ITD was also identified. A diagnosis of acute promyelocytic leukemia was made.

\section{Treatment}

Given the diagnosis of APL, the patient was urgently admitted to the hospital to begin treatment with ATRA and chemotherapy. Induction was started with idarubicin $12 \mathrm{mg} / \mathrm{m}^{2}$ (days 2, 4, $6,8)$ and ATRA (AIDA regimen). ${ }^{10}$ His course

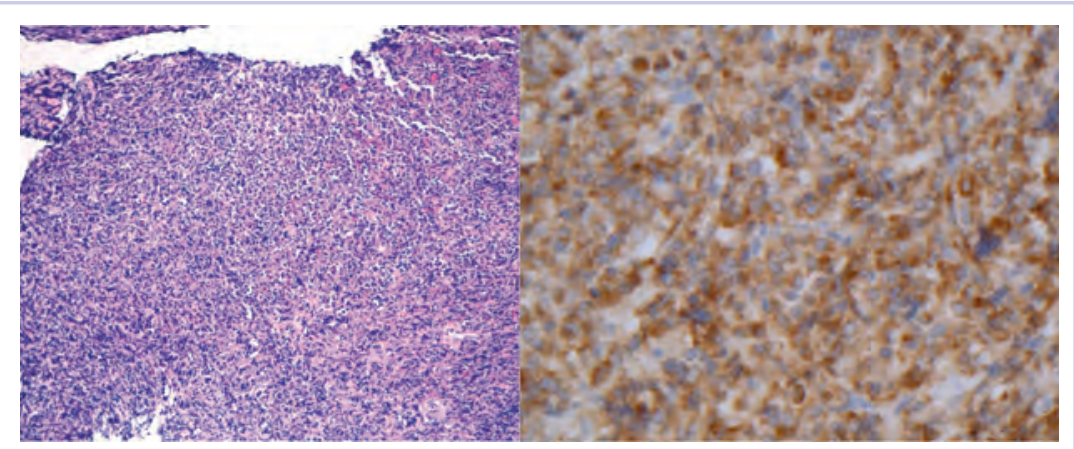

FIGURE 2 Paraspinal mass biopsy. A, Left, H\&E stain; B, Right, myeloperoxidase stain.

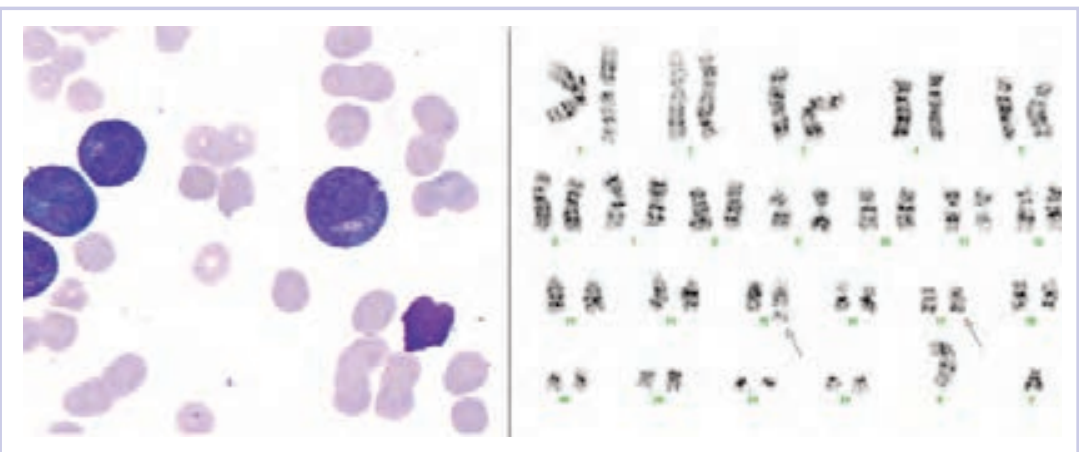

FIGURE 3 Bone marrow biopsy. A, Left aspirate smear; B, Right cytogenetics. was complicated by differentiation syndrome responsive to steroids, E. coli sepsis, C. difficile colitis, and presumed fungal pneumonia. He did not experience any coagulopathic complications. A repeat bone marrow biopsy after induction demonstrated a normocellular marrow with no morphologic evidence of residual APL. A positron-emission tomography-computed-tomography scan performed to evaluate his T7 lesion was negative. Given his normal white blood cell and platelet counts at presentation, the patient was stratified as low risk, despite his extramedul- lary disease. Accordingly, the patient was planned to receive 3 cycles of anthracycline-based consolidation as per the AIDA regimen. However, subsequent cardiac evaluations revealed a drop in the patient's ejection fraction and consequently he received consolidation with ATRA-ATO for 7 months. He completed therapy in July 2013 and was in a molecular remission on bone marrow evaluation at that time.

A year after remission, the patient developed molecular relapse peripherally. Concurrent bone marrow biopsy 
revealed the $\mathrm{t}(15 ; 17)$ by cytogenetics but no evidence of morphologic relapse. A repeat MRI scan of his spine was negative for relapsed extramedullary disease. The patient received salvage ATO with progressive cytopenias, and a repeat marrow study showed progression to overt morphologic relapse. He was then treated with high-dose cytarabine (HiDAC) induction chemotherapy and achieved a second morphologic and molecular remission at the time of his recovery marrow. He received an additional cycle of HiDAC chemotherapy with granulocyte-colony stimulating factor mobilization for autologous stem-cell collection. He underwent a busulfan-cyclophosphamide-conditioned autologous transplant and is now in an ongoing remission 3 months after the transplant.

\section{Discussion}

This case represents an atypical extramedullary presentation of APL as a paraspinal mass. Extramedullary disease is seen in fewer than $10 \%$ of AML cases, and occurs more frequently in cases with (myelo)monocytic morphology and in AML with $\mathrm{t}(8 ; 21)(\mathrm{q} 22 ; \mathrm{q} 22){ }^{6,11}$ Granulocytic sarcoma (also referred to as myeloid sarcoma or extramedullary AML) is a manifestation of a myeloid leukemia in which the neoplastic myeloid cells form an extramedullary tumor mass composed of immature myeloid cells, and can develop de novo, concurrently with medullary AML, or at relapse. ${ }^{12,13}$ The exact frequency of APL presenting this way at diagnosis is unknown as such presentations are reported only in isolated case reports or case series. ${ }^{14}$

APL is a molecularly defined entity with a specific treatment and favorable prognosis. The clinical presentation seen here is unusual in a number of respects. First, although APL has been known to manifest as granulocytic sarcoma, this most typically occurs in the setting of prior treatment, possibly related to prior use of ATRA..$^{7-9}$ In our patient with a new diagnosis of APL, extramedullary disease is an exceedingly rare occurrence. Second, most patients with untreated APL develop cytopenias with a rapidly progressive clinical course, but our patient had a 4-month history of back pain before seeking medical attention. Furthermore, despite the mass, the patient had preserved blood counts with normal coagulation parameters, which is inconsistent with this generally aggressive acute leukemia. Third, our patient was deemed to have low-risk disease by traditional measures such as white blood cell and platelet counts based on current risk stratification. ${ }^{3,15}$ Although low-risk patients are generally felt to have a favorable prognosis, our patient with extramedullary disease relapsed shortly after completing his initial therapy, suggesting that this presentation may be consistent with a higher risk than APL patients without extramedullary disease at diagnosis. Despite his poor response to salvage ATO, our patient is now in a second remission following HiDAC chemotherapy and has been able to undergo a consolidative autologous transplant.

\section{Conclusion}

APL is a subtype of acute myeloid leukemia that is defined by the PML-RARA $t(15 ; 17)$ translocation. Although historically an aggressive disease with high mortality rates, molecularly targeted therapies have revolutionized the treatment of this leukemia and have made it one of the great successes in cancer therapy. In this case, we describe an unusual and rare presentation of APL as a paraspinal mass. Although the morphological findings seen in the bone marrow and paraspinal mass were not entirely prototypic, genetic analyses performed on the bone marrow identified a $\mathrm{t}(15 ; 17)(\mathrm{q} 24 ; \mathrm{q} 12)$, underlining the significance of a complete evaluation in all patients with acute leukemia prior to initiation of therapy. Furthermore, though the patient was stratified as low risk by standard measures, his clinical course was consistent with a more aggressive disease and it remains unclear if extramedullary involvement at presentation correlates with higher risk APL. Given the rarity of this situation, there will likely not be a standardized approach for APL with granulocytic sarcoma, highlighting the importance of reporting individual cases.

\section{Acknowledgment}

For corresponding author: fellowship funding support via Cancer Center Research Training Program: NCI 5-T32 CA09615-25.

\section{References}

1. Wang Z-Y, Chen Z. Acute promyelocytic leukemia: from highly fatal to highly curable. Blood. 2008;111:2505-2515.

2. Tallman MS, Altman JK. How I treat acute promyelocytic leukemia. Blood. 2009;114:5126-5135.

3. Coombs CC, Tavakkoli M, Tallman MS. Acute promyelocytic leukemia: where did we start, where are we now, and the future. Blood Cancer J. http://www.ncbi.nlm.nih.gov/pmc/articles/PMC4450325/. Published April 17, 2015. Accessed February 8, 2016.

4. Lo-Coco F, Avvisati G, Vignetti M, et al. Retinoic acid and arsenic trioxide for acute promyelocytic leukemia. N Engl J Med. 2013;369:111-121.

5. Iland HJ, Bradstock K, Supple SG, et al. All-trans-retinoic acid, idarubicin, and IV arsenic trioxide as initial therapy in acute promyelocytic leukemia (APML4). Blood. 2012;120:1570-1580; quiz 1752.

6. Bakst RL, Tallman MS, Douer D, et al. How I treat extramedullary

acute myeloid leukemia. Blood. 2011;118:3785-3793.

7. Vega-Ruiz A, Faderl S, Estrov Z, et al. Incidence of extramedullary disease in patients with acute promyelocytic leukemia: a single-institution experience. Int J Hematol. 2009;89:489-496.

8. de Botton S, Sanz MA, Chevret S, et al. Extramedullary relapse in acute promyelocytic leukemia treated with all-trans retinoic acid and chemotherapy. Leukemia. 2006;20:35-41.

9. Albano F, Specchia G Extramedullary disease in acute promyelocytic leukemia: two-in-one disease. Mediterr J Hematol Infect Dis. http://www.ncbi.nlm.nih.gov/pmc/articles/PMC3248343/. Published December 21, 2011. Accessed February 8, 2016.

10. Lo-Coco F, Avvisati G, Vignetti M, et al. Front-line treatment of acute promyelocytic leukemia with AIDA induction followed by risk-adapted consolidation for adults younger than 61 years: results of the AIDA-2000 trial of the GIMEMA Group. Blood. 
2010;116:3171-3179.

11. Tallman MS, Hakimian D, Shaw JM, et al. Granulocytic sarcoma is associated with the 8;21 translocation in acute myeloid leukemia.J Clin Oncol. 1993;11:690-697.

12. Campidelli C, Agostinelli C, Stitson R, et al. Myeloid sarcoma: extramedullary manifestation of myeloid disorders. Am J Clin Pathol. 2009;132:426-437.

13. Pileri SA, Ascani S, Cox MC, et al. Myeloid sarcoma: clinicopathologic, phenotypic and cytogenetic analysis of 92 adult patients.
Leukemia. 2007;21:340-350.

14. Pacilli L, Lo Coco F, Ramadan SM, et al. Promyelocytic sarcoma of the spine: a case report and review of the literature. Adv Hematol. http://dx.doi.org/10.1155/2010/137608. Published 2010. Accessed February 8, 2016.

15. Sanz MA, Martín G, González M, et al: Risk-adapted treatment of acute promyelocytic leukemia with all-trans-retinoic acid and anthracycline monochemotherapy: a multicenter study by the PETHEMA group. Blood. 2004;103:1237-1243. 